\title{
The Meaning Function Four Dimensional View: Procedural Approach
}

\author{
Hatem El-Ssayed ${ }^{1}$, Aly N. Elwakeil ${ }^{2,3}$ \\ ${ }^{1}$ Cleveland State University, Cleveland, USA \\ ${ }^{2}$ Banha University, Banha, Egypt \\ ${ }^{3}$ Alobour Higher Institute for Management, Computer Science and Informatics, Cairo, Egypt \\ Email: hat_m74@yahoo.com
}

How to cite this paper: El-Ssayed, H., \& Elwakeil, A. N. (2018). The Meaning Function Four Dimensional View: Procedural Approach. Open Journal of Modern Linguistics, 8, 23-38.

https://doi.org/10.4236/ojml.2018.82004

Received: February 18, 2018

Accepted: April 27, 2018

Published: April 30, 2018

Copyright ( 92018 by authors and Scientific Research Publishing Inc. This work is licensed under the Creative Commons Attribution International License (CC BY 4.0).

http://creativecommons.org/licenses/by/4.0/

(c) (i) Open Access

\begin{abstract}
This paper proposes a new procedural model for the meaning theory as a vector valued function. This function exhibits the consistency and isomorphism properties through the vector space. The new model explains the procedural mechanism of the lingual communication between the speaker and the audience in four-dimensional view. This approach can lead to several applications in different fields. The encoding of texts or speech by the useful properties of vector spaces and their linearity property will improve the digital translation quality. This consequently will improve the precession of the statistical and computational analysis of texts. Programming the statements of a text will be established easily. This in turn can be a subject matter of the decision-making in general and informatics. In addition, the model gives a clear distinction between the two terms "explanation" and "interpretation" of a specific text. Furthermore, the model feeds the perception of how the meaning appears in different dimensions based on the signification vectors which can be constructed according to the meaning state of a given text. This has been a big dilemma in the linguistic academic field long time ago.
\end{abstract}

\section{Keywords}

Computational Linguistics, Vector Analysis, Surface Integral, Meaning Theory

\section{Introduction}

The meaning function has been an important target of study by scholars and scientists all over the human intellectual history. It possessed the interest of a huge number of scholars who belong to different backgrounds such as: philosophy, linguistics, psychology, and mathematics. The authors in the current research 
paper are introducing a new numerical model postulating the meaning theory in four-dimensional view. On one hand, this approach will be dealing with the meaning perspective as a continuous function and concerning the speech itself abstractly as a vector field.

On the other hand, this will enhance and even create new practical applications in different scientific fields such as but not limited: computational linguistics, psychiatric linguistics, IQ intelligence test for intelligent evaluation credibly, robot and call centers industrial technology. In addition, the useful applications of that model in the analysis and encoding texts will make a huge jump in stock marketing four dimensional analyses.

Lakoff \& Turner (1989) studied the metaphoric language and they concluded that it is a structure which is coming from a different conceptual domain. The computational approach introduced by Fass (1991) was an entrance for the application of vectors in recognizing the metaphors and metonymies in a text. Cook and Stevenson (2010) continued the computational view automatically by detecting semantic changes with time.

A vector function of one real variable is a function which has an interval of real numbers $\mathrm{I}=[a, b] \subset \mathbb{R}$ as a domain and its range is a set of $n$-tuples of real numbers (Stewart, 1999).

The three-dimensional vector function which the authors use in this paper fulfils the condition that for each number $t$ in the domain of $(\hat{\boldsymbol{r}})$ there exists a unique vector $\hat{\boldsymbol{r}}(t)$ in three dimensions. If $x(t), y(t)$, and $z(t)$ are the component functions of the vector $\hat{\boldsymbol{r}}(t)$ or the coordinates of the vector in the Cartesian coordinate system. We can write the vector function in the parametric form as follows:

$$
\hat{\boldsymbol{r}}(t)=(x(t), y(t), z(t))=x(t) \hat{\boldsymbol{i}}+y(t) \hat{\boldsymbol{j}}+z(t) \hat{\boldsymbol{k}}, t \in \mathrm{I}
$$

where $\hat{\boldsymbol{i}}, \hat{\boldsymbol{j}}$ and $\hat{\boldsymbol{k}}$ are respectively unit vectors in the directions of $X, Y$, and $Z$; the three main axes of the Cartesian coordinate system.

Hirtle (1985) stated that for linguistics to achieve its goal, we have to study a human language in all its dimensions. The dimensions were mentioned as:

1) The class of the language, whether the language is belonging to the Indo-European group of languages or other types.

2) The temporal dimension; this includes the diachronic and synchronic analysis.

3) The existential dimension which considers the mental and physical approaches in a language.

4) The operational dimension which provides how a word and a sentence were constructed in a sentence.

Fauconnier \& Turner (2002) discussed in detail how our minds and even animal minds are able to visualize or estimate the significance of different objects or events occurring (can be seen, or can be heard simultaneously). They introduced the blending technique which became a useful technique in studying the metaphor. By blending, the mind can integrate non-occupying objects or even 
simultaneous events successfully.

Gagliano et al. (2016) made a practical progress by using the blending technique to explain the semantic view of the figurative language. They related the semantic space domain of the metaphor to its original ancestor: the vector of an anchor word and the related vector of a poetic theme. According to them, the blending between those vectors will create the metaphoric meaning.

\section{Construction of the Meaning Function Model}

The meaning function $(\boldsymbol{M})$ is a vector field on $\mathbb{R}^{3}$ (see Figure 1 ).

It can be expressed in terms of its component functions $(x, y$, and $z)$ as follows:

$$
\boldsymbol{M}(x, y, z)=x \hat{\mathbf{i}}+y \hat{\mathbf{j}}+z \hat{\boldsymbol{k}}
$$

where:

“ $X$ " is the component of the morphological level of a specific word with any human language,

" $y$ " represents the component of the syntax or the set of rules by which we can put the words together to create a logical meaning, $j$,

" $z$ " is the component of the rhetorical styles in a language which coincide with the situation of the speech.

$\hat{\boldsymbol{i}}, \hat{\boldsymbol{j}}$ and $\hat{\boldsymbol{k}}$ are respectively unit vectors in the directions of $X, Y$, and $Z$ (the main Cartesian axes). The continuity of $M$ can be easily defined due to the continuity of its components inside the local body of a community.

The gradient vector $\nabla \boldsymbol{M}$ of the meaning function is:

$$
\nabla \boldsymbol{M}=\frac{\partial M}{\partial x} \hat{\boldsymbol{i}}+\frac{\partial M}{\partial y} \hat{\boldsymbol{j}}+\frac{\partial M}{\partial z} \hat{\boldsymbol{k}}
$$

\subsection{The Dictionary Meaning of Words in the Simple One Dimensional Case}

In the ideal case, the meaning function $\boldsymbol{M}(x, y, z)$ fulfils the dimensional-consistency and it is continuous in the three dimensional space. How can we

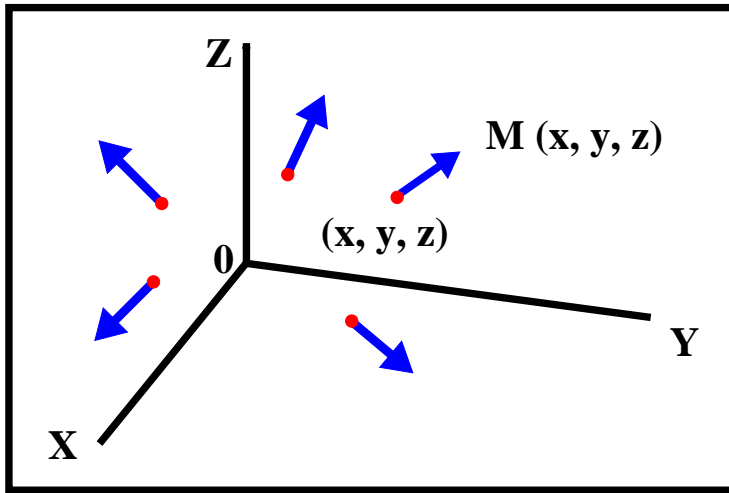

Figure 1. The meaning function as a vector field on $\mathbb{R}^{3}$ (adapted from Stewart, 1999). 
represent the coordinate values of the meaning function in terms of numbers and expressions? That was a hard lingering question but the answer can be approached closely through the following lines.

On the morphological level, the meaning component $x$ is the number of words in a complete sentence or equals one if we are referring to the dictionary-meaning of a word specifically. For example, if we want to write a meaning vector component which is related to the word "answer," where $(x=1)$. We will have:

$$
\boldsymbol{M}=\langle 1,0,0\rangle=1 \hat{\boldsymbol{i}}+0 \hat{\mathbf{j}}+0 \hat{\boldsymbol{k}}
$$

If we try to represent the word "really" with no intonation factor, Equation (3) would be the perfect form. When the intonation "really!" comes along as an exclamation reaction of the audience, it will introduce the variable $x$ to the component of $M$ along the $x$-axis and Equation (3) will become:

$$
\boldsymbol{M}=\langle 1+x, 0,0\rangle=(1+x) \hat{\boldsymbol{i}}+\hat{\boldsymbol{j}}+0 \hat{\boldsymbol{k}}
$$

It is clear that the one-dimensional meaning function $M_{X}$ will construct a linear relation and can be represented by an equation of a line as: $M_{x}=m x+b$; where $m$ is the slope of the line and $b$ is the $y$-intercept and at the same time equals the number of the dictionary words, Figure 2.

\subsection{The two Dimensional Case of the Meaning Function}

In the two-dimensional case, the syntax or the grammar of a human language will contribute a supporting level into the meaning function. The sentence here will have either a regular structure form, i.e. an active form, or a deviated normal form but is still meaningful, such as: the passive voice, the indirect speech, and moving the words forward or delaying them. In this case any simple normal structural form of a sentence will be assigned the value one.

Let us give an example of simple two-dimensional case. The sentence "the cat is at the house" is a complete sentence. It has a subject, a verb and a complement respectively. It can be written mathematically as follows:

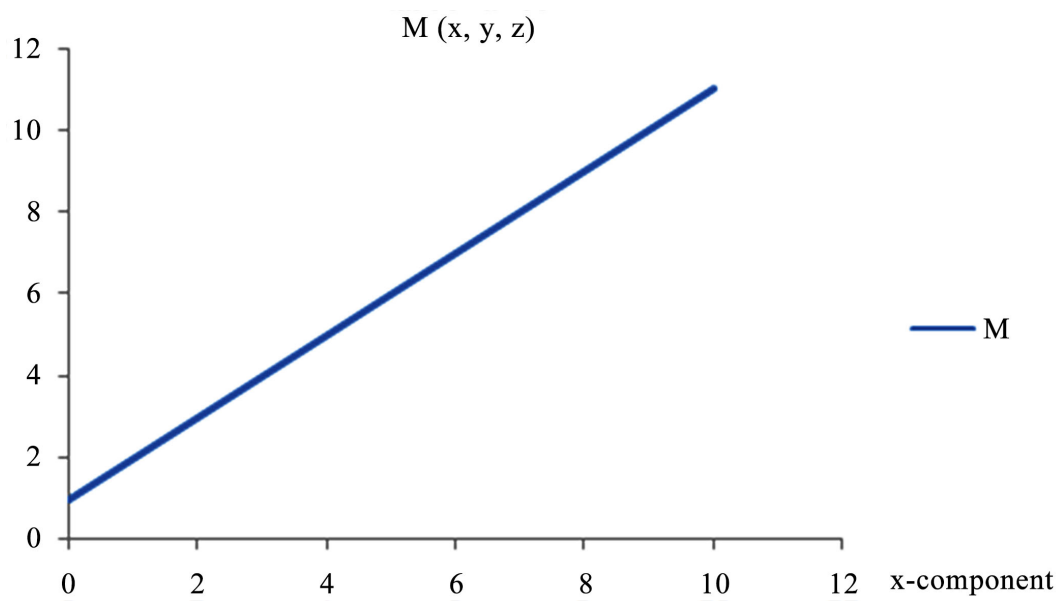

Figure 2. The component of the meaning function in the direction of $\mathrm{X}$-axis. 


$$
\boldsymbol{M}_{i, j}=\langle 6,1+y, 0\rangle=(6) \hat{\boldsymbol{i}}+(1+y) \hat{\boldsymbol{j}}+0 \hat{\boldsymbol{k}}
$$

In this equation, the value of the second coordinate is $(1+y)$. The number one refers to the number of structures which appeared in the sentence and the variable " $y$ " represents the type of structure along the sentence. When $y=0$ there is no deviation than the normal structure. Moving up into a more complicated form of a sentence, the variable $y$ will be an outstanding factor in the second component estimation of the meaning function.

A new sentence "the cat was left at the house" induces in the audience mind two facts; the first is: the "cat is at the house" and the second is: "the cat was being at the house by mistake". Definitely, any change here in the second component's value will appear in Equation (5), so, it will look like:

$$
\boldsymbol{M}_{i, j}=\langle 7+x, 2+y, 0\rangle=(7+x) \hat{\boldsymbol{i}}+(1+y) \hat{\boldsymbol{j}}+0 \hat{\boldsymbol{k}}
$$

where the variable $y$ represents any change in the structure of a given sentence. Again, the presence of the intonation factor added and enhanced the meaning in the mind of the audience. The sentence "the cat was left at the house" when applying intonation will let the audience feel that the cat could be in trouble because of the lack of food or the cat should have been with us to see the veterinarian. In Equation (6), $x=2$ because the speaker applied the intonation twice. Here, the meaning function grows up more and the $y$-component of the meaning function takes the straight line shape, Figure 3.

\subsection{The Three Dimensional Case of the Meaning Function}

The existence of the rhetorical tools in the text will move up or down the other two dimensional meaning components adding a new dimension, the 3rd dimension, acting as a height or an amplitude for the two-dimensional case. We can illustrate that by analyzing the following sentence as a vector function: "the cat had asked me its food before it was left at the house." The listener will get the meaning in three dimensions. He will realize that there is a cat and that cat was left at the house by mistake. In addition, that cat is in trouble because she was hungry and she behaved as a human or a kid metaphorically. The cat requested its food, so, the cat could be in trouble.

The meaning function here will exhibit the third component which is the rhetorical one. The existence of that component has a great effect on the meaning function by drawing a complete image with high resolution. We approximate the metaphor or the metonymy here as an exponential function $Z=\left(e^{z}\right)$ due to the huge effect in enhancing the meaning image in the audience mind. It is clear that the third component grows up when it is compared with the second one (the quadratic form of the Y-direction), Figure 4.

Then the meaning function $\boldsymbol{M}(x, y, z)$ in three dimensions can be formulated in the vector form as follows:

$$
\boldsymbol{M}_{i, j, k}=\left\langle 14+x, 2+y, \mathrm{e}^{z}\right\rangle=(14+x) \hat{\boldsymbol{i}}+(2+y) \hat{\boldsymbol{j}}+\mathrm{e}^{z} \hat{\boldsymbol{k}}
$$




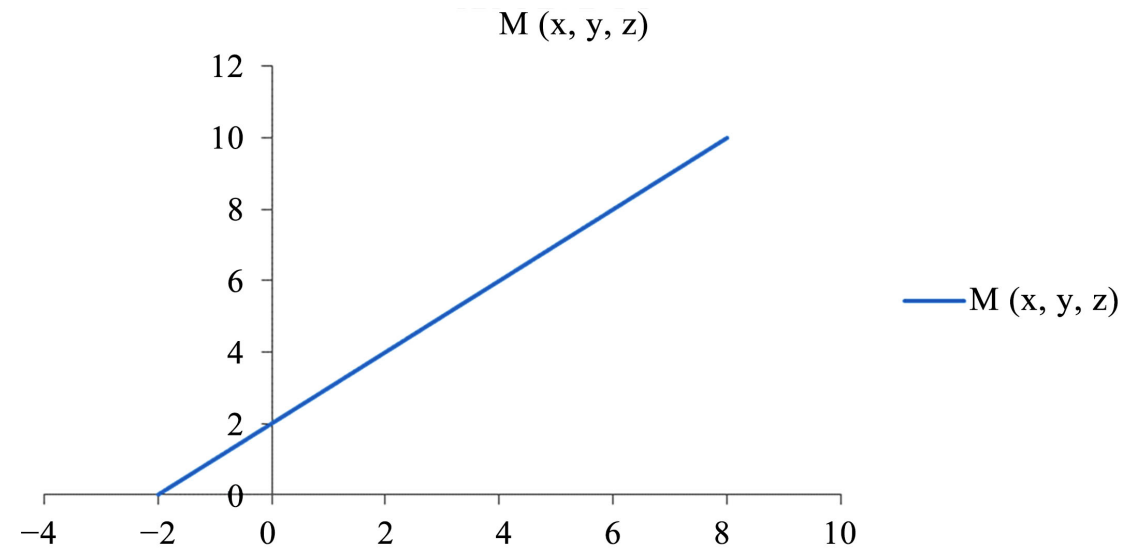

Figure 3. The component of the meaning function in the direction of $\mathrm{Y}$-axis.

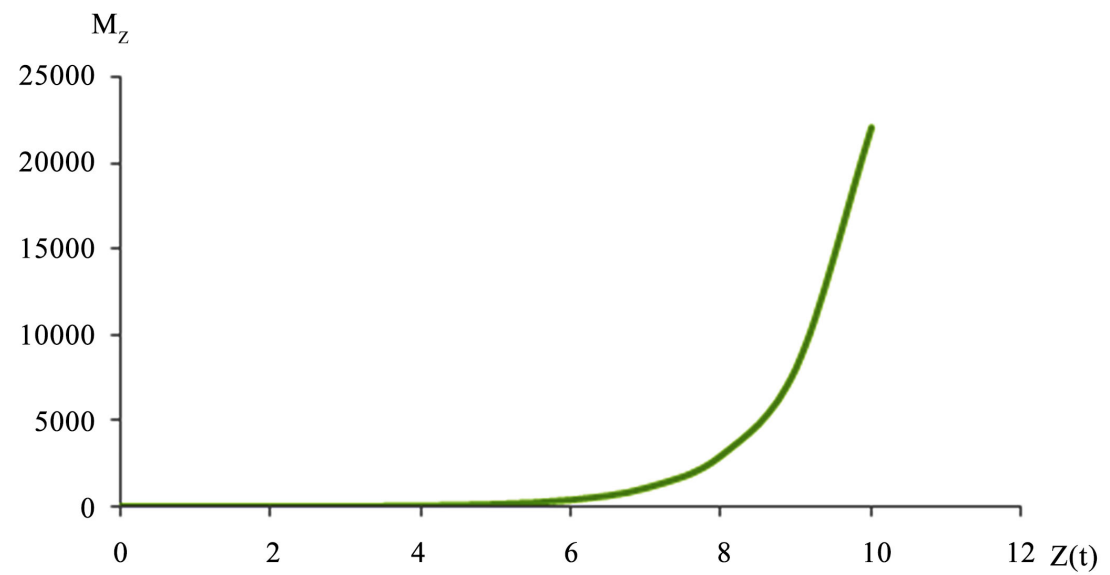

Figure 4. The component of the meaning function in the Z-direction.

where:

$(14+x)$ is the component of the meaning function in the $\mathrm{X}$-direction which is represented by 14 words and 3-intonation syllabi.

$(2+y)$ is the structure of the compound system which includes two sentences were conjugated by "before" and the passive voice variable $y$ which appears one time in the sentence. This is considered as the component of the meaning function in the Y-direction. $\left(e^{z}\right)$ is the meaning function component in the Z-direction which represents the metaphoric level.

\subsection{The Meaning Function: 4-Dimensional Approach}

Coming back to Equation (7), the dilemma of the metaphor and the metonymy problem holds our model to be accepted and makes it very difficult or not easy to be understood. The analysis of the metaphor leads us to add a binary relationship between two objects; these objects cannot occupy and each of them has its own attributes, Fass (1991). For example, when someone said the following statement "I was talking to a lion yesterday evening in the train." In this sentence, the speaker is describing a strong man and he was talking to him in the train. The listeners fast enough to get that the speaker-man is not crazy and he 
was not talking to a real lion yesterday in the train.

When the audience has enough intelligence and awareness of the language styles, they will be able to understand the speaker's figurative speech and it was approved from the audience inside the native speaker's community body set in a certain area. The one-to-one map between the two objects (the lion-the man) enables one of the objects to give the other all or some of his attributes and vice versa without any occupation (see Figure 5).

We could ask a series of questions now: Where can this attributes-trading-process happen? And how? Two good questions need answers.

The function $\boldsymbol{M}(x, y, z)$ ranged from zero speech (silence state) into the maximum positive absolute eloquence level having the maximum value. This value is the value on the border between the imagination and the closed 3-d region of meaning; when the speaker decorates his speech by introducing the metaphors and the metonymies in addition to the other rhetorical tools. This is the affirmation case. In the negation case, that value will be assigned the negative sign. The $\mathrm{x}, \mathrm{y}$, and $\mathrm{z}$ variables are approaching the maximum outer border of the meaning body and become closed enough to the invisible imagination power of the fourth-dimension.

Of course, we cannot see the $4^{\text {th }}$ dimension spatially although it does exist. It will be invisible for our mind because the latter is able to comprehend through the world in which we live. Fortunately, we can compare that $4^{\text {th }}$ dimension with the $3^{\text {rd }}$ one and hence we can notice the effect of that dimension on the intersection area. In other words, when the speaker reaches the abnormal state of eloquence, he will start to exaggerate the attributes of real objects. Here, the imaginative power (invisible dimension) will be in contact to the surface of the sphere of meaning which in turn includes the three visible other dimensions (see Figure 6).

Here, in this state a dimensional gate between the $3^{\text {rd }}$ and the $4^{\text {th }}$ dimensions will be opened. This gate enables the lion and the man in our example or the cat and the kid in the above example to trade their specific attributes. When the zone of the figurative language dumped down, the meaning function comes back to its original form as three-dimensional vector function.

The metaphors will force us to rectify our model for the meaning function to be a fourth dimensional vector function and each statement can be represented by 4 -dimensional meaning vector function. In the absence of the figurative language, we assign the value of the fourth dimensional component the number zero. Hence, Equation (7) will be written in the vector form as:

$$
\boldsymbol{M}_{i, j, k, l}=\left\langle 14+x, 2+y, \mathrm{e}^{z}, 1\right\rangle=(14+x) \hat{i}+(2+y) \hat{\boldsymbol{j}}+\mathrm{e}^{z} \hat{\boldsymbol{k}}+(1) \hat{\boldsymbol{i}}
$$

where $\boldsymbol{q}=$ (1) represents the vector component of the imagination power of the $4^{\text {th }}$ dimension in the direction of the unit vector $\hat{\boldsymbol{l}}$ in relation to the vertical $\mathrm{S}$-coordinate axis, i.e. when the figurative language exists in the speech.

\subsection{The Meaning Sphere as a 4-D Model}

We discussed above how simply we can formulate mathematically the statements 


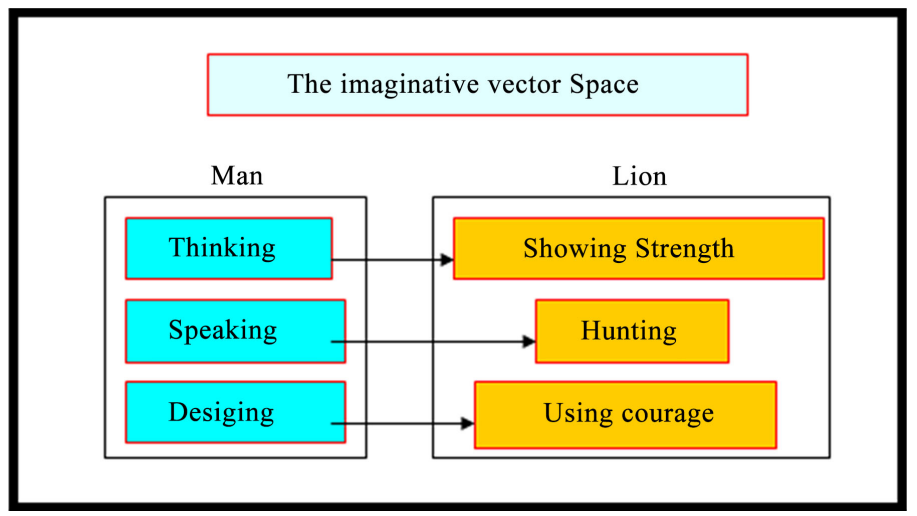

Figure 5. The binary relation one-to-one imaging in the imagination vector space of the human mind.

The imaginative Power The intersection between the $3 \mathrm{rd}$ and the 4 th dimensions

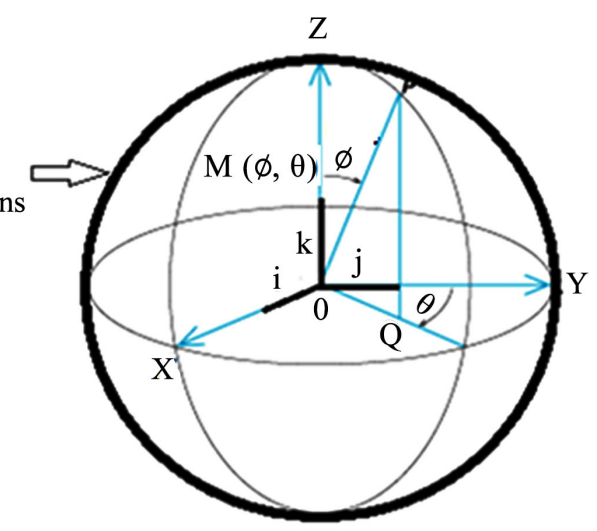

Figure 6. The imaginative power as an intersection between the third and the fourth dimensions (adapted from, Science Wiki, 2017).

of a given text. The text is a collection of statements in the space containing it and was replaced here in our model by vectors. These vectors can be linearly dependent and that will strengthen the meaning stream in a certain direction or they can be linearly independent with discontinuity of the meaning stream, Figure 7.

The human mind can analyze the language text and can check the linear independence between these meaning vectors producing the sense of continuity or discontinuity of meaning. Figure 7 (a) shows the geometry of two vectors representing two statements in one text. The vector $V_{1}$ equals the statement "the man was running fast," while vector $V_{2}$ equals the statement "the man arrived on time into his work." These two vectors are linearly dependent. Figure 7 (b) illustrates another case. Vector $V_{1}$ equals the statement "the sky is clear" and $V_{2}$ equals the statement "it is cloudy" in the same text are linearly independent.

As we mentioned above, the text in our model is a collection of vectors and these vectors obey the axioms of the vector space and exhibits all its linearity properties. The text is also a conservative vector field function in four-dimensions. The usage of the surface integral with a sphere as a closed-surface will encode the meaning stream in the targeting text. 


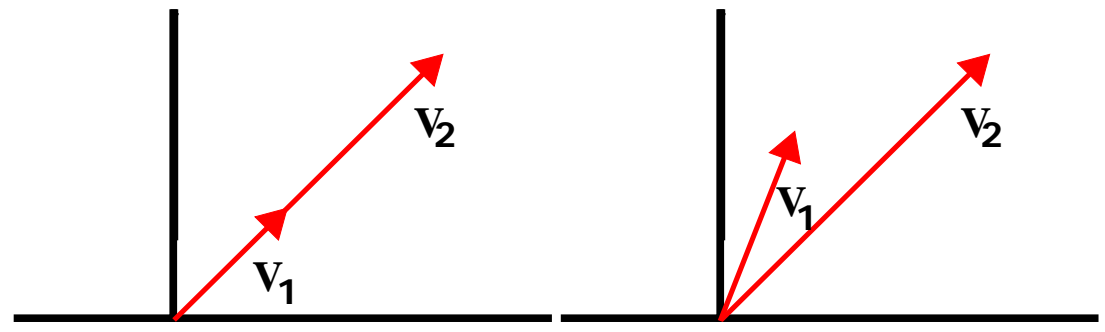

(a)

Figure 7. Linear independence: (a) the vectors $V_{1}$ and $V_{2}$ are linearly dependent in the same text; (b) The vectors $V_{1}$ and $V_{2}$ are linearly independent in the same text (adapted from, Leon, 2006).

The flux of meaning across the sphere is:

$$
\mathrm{MF}=\iint_{\mathrm{T}} \boldsymbol{M} \cdot \mathrm{d} \boldsymbol{T}
$$

where $T$ represents the surface of a given sphere in the parametric form by using the vector function:

$$
\hat{\boldsymbol{r}}(\phi, \theta)=x(\phi, \theta) \hat{\boldsymbol{i}}+y(\phi, \theta) \hat{\boldsymbol{j}}+z(\phi, \theta) \hat{\boldsymbol{k}}
$$

The equation of a sphere is:

$$
x^{2}+y^{2}+z^{2}=a^{2}
$$

when we describe the values of $(x, y, z)$ in Equation (10) by using spherical coordinates, we will obtain: $x=a \sin \phi \cos \theta, y=a \sin \phi \sin \theta, \quad z=a \cos \phi$. Then, Equation (10) can be written as:

$$
\begin{aligned}
& \hat{\boldsymbol{r}}(\phi, \theta)=(a \sin \phi \cos \theta) \hat{\boldsymbol{i}}+(a \sin \phi \sin \theta) \hat{\boldsymbol{j}}+a \cos \phi \hat{\boldsymbol{k}}, \\
& \mathrm{D}=\{(\theta, \phi) \mid \theta \in[0,2 \pi], \phi \in[0, \pi]\}
\end{aligned}
$$

$\mathrm{D}$ is the parameters' domain. Since the sphere is a smooth oriental surface, then it is instinctively provided with the orientation of the unit normal vector $\hat{\boldsymbol{n}}$ which is positive in our case.

$$
\hat{\boldsymbol{n}}=\frac{\boldsymbol{r}_{\phi} \times \boldsymbol{r}_{\theta}}{\left\|\boldsymbol{r}_{\phi} \times \boldsymbol{r}_{\theta}\right\|}
$$

For the sphere in Equation (11), we have:

$$
\boldsymbol{r}_{\phi} \times \boldsymbol{r}_{\theta}=\left(a^{2} \sin ^{2} \phi \cos \theta\right) \hat{\boldsymbol{i}}+\left(a^{2} \sin ^{2} \phi \sin \theta\right) \hat{\boldsymbol{j}}+\left(a^{2} \sin \phi \cos \theta\right) \hat{\boldsymbol{k}}
$$

and

$$
\left\|\boldsymbol{r}_{\phi} \times \boldsymbol{r}_{\theta}\right\|=a^{2} \sin \phi
$$

The value of $\hat{\boldsymbol{n}}$ in Equation (13) will be $\frac{1}{a} \hat{\boldsymbol{r}}(\phi, \theta)$.

Finally, Equation (9) can be written as:

$$
\mathrm{MF}=\iint_{\mathrm{T}} \boldsymbol{M} \cdot \mathrm{d} \boldsymbol{T}=\iint_{\mathrm{D}} \boldsymbol{M} \cdot\left(\boldsymbol{r}_{\phi} \times \boldsymbol{r}_{\theta}\right) \mathrm{d} A
$$

where $T$ is the surface of the sphere and D is the parameter domain. Now, we can evaluate the Meaning Flux (MF) of the sphere by using the positive orienta- 
tion of the field across the whole surface of the sphere. The sphere surface $\mathrm{T}$ is a closed surface and we can use Equation (16) with D, being the projection of both the upper hemisphere $\left(\mathrm{MF}_{\mathrm{Up}}\right)$ and the projection of the lower hemisphere $\left(\mathrm{MF}_{\mathrm{Lo}}\right)$ on the $x y$-plane which is the equatorial disk $x^{2}+y^{2} \leq a^{2}, z=0$.

The meaning flux of any text can be estimated in the same way as in Equation (16), and Equation (8) can be expanded to include the rest of the rhetorical tools which the speaker is using to capture the attention of the audience. It is relevant here to mention that there is an obligation condition. This condition is that the audience has to have the same semantic tools to receive the whole speaker's intended meaning beyond his speech. Any deficiency in this system will lead to a partial transfer of the ideas conveyed between the speaker and the listeners. Consequently, the procedural semantic system will exhibit lack of equilibrium.

Since the sphere have eight octants, and then the vectors of the text can belong to anyone of them. Table 1 illustrates eight vectors related to the eight octants of the meaning sphere. The same example has been used to avoid any confusion. We can see the reliability of the new model and it is the flexibility inside the metric space side by side to the useful linear properties of the vector space.

This model enables us to re-write any text as a set of vectors which will make the semantic analysis of a text very easy. The vectors which were illustrated in the tables take the following numerical forms:

$$
\begin{aligned}
& \boldsymbol{V}_{1}=\left(\begin{array}{c}
14+x \\
2+y \\
\mathrm{e}^{z} \\
1
\end{array}\right), \boldsymbol{V}_{2}=\left(\begin{array}{c}
(-1)(14+x) \\
2+y \\
\mathrm{e}^{z} \\
1
\end{array}\right), \boldsymbol{V}_{3}=\left(\begin{array}{c}
(-1)(14+x) \\
(-1)(2+y) \\
\mathrm{e}^{z} \\
1
\end{array}\right), \boldsymbol{V}_{4}=\left(\begin{array}{c}
14+x \\
(-1)(2+y) \\
\mathrm{e}^{z} \\
1
\end{array}\right) \\
& \boldsymbol{V}_{5}=\left(\begin{array}{c}
14+x \\
(-1)(2+y) \\
(-1)\left(\mathrm{e}^{z}\right) \\
-1
\end{array}\right), \boldsymbol{V}_{6}=\left(\begin{array}{c}
(-1)(14+x) \\
(-1)(2+y) \\
(-1)\left(\mathrm{e}^{z}\right) \\
-1
\end{array}\right), \boldsymbol{V}_{7}=\left(\begin{array}{c}
14+x \\
2+y \\
(-1)\left(\mathrm{e}^{z}\right) \\
-1
\end{array}\right), \boldsymbol{V}_{8}=\left(\begin{array}{c}
(-1)(14+x) \\
2+y \\
(-1)\left(\mathrm{e}^{z}\right) \\
-1
\end{array}\right)
\end{aligned}
$$

We can apply the vector properties on these vectors easily. For example, we can add the vector $V_{1}$ to the vector $V_{6}$ which represents the negation of the sentence in $V_{1}$ and we will get the vector 0 (the addition identity in vector space). It is obvious now that we can apply the whole linearity properties of the vector space into any text.

$$
\boldsymbol{V}_{1}=\left(\begin{array}{c}
14+x \\
2+y \\
\mathrm{e}^{z} \\
1
\end{array}\right)+\boldsymbol{V}_{6}=\left(\begin{array}{c}
(-1)(14+x) \\
(-1)(2+y) \\
(-1)\left(\mathrm{e}^{z}\right) \\
-1
\end{array}\right)=\mathbf{0}=\left(\begin{array}{l}
0 \\
0 \\
0 \\
0
\end{array}\right)
$$

\subsection{Case Study}

By taking $a=1$, we are dealing with a unit sphere for the purpose of simplification of the calculation. Furthermore, by plugging the value of $\boldsymbol{M}$ from Equation (8) into Equation (16) we will have: 
Table 1. Vectors signs according to their positions in the cartesian octants.

\begin{tabular}{|c|c|c|c|c|c|}
\hline & The vector of the sentence & $\mathrm{X}$-axis & Y-axis & Z-axis & Octant No. \\
\hline 1) & $\begin{array}{l}\text { The cat had asked me its food before it } \\
\text { was left at the house. }\end{array}$ & Positive & Positive & Positive & First \\
\hline 2) & $\begin{array}{l}\text { The (not cat) had asked me its food } \\
\text { before it was left at the house. }\end{array}$ & Negative & Positive & Positive & Second \\
\hline 3) & $\begin{array}{l}\text { The (not cat) had (not) asked me its } \\
\text { food before it was left at the house. }\end{array}$ & Negative & Negative & Positive & Third \\
\hline 4) & $\begin{array}{l}\text { The (cat) had (not) asked me its food } \\
\text { before it was left at the house. }\end{array}$ & Positive & Negative & Positive & Fourth \\
\hline 5) & $\begin{array}{l}\text { The cat had (not) asked me its food } \\
\text { before it was (not) left at the house. }\end{array}$ & Positive & Negative & Negative & Fifth \\
\hline 6) & $\begin{array}{l}\text { The (not cat) had (not) asked me its } \\
\text { food before it was (not) left at the house. }\end{array}$ & Negative & Negative & Negative & Sixth \\
\hline 7) & $\begin{array}{l}\text { The cat had asked me its food before it } \\
\text { was (not) left at the house. }\end{array}$ & Positive & Positive & negative & Seventh \\
\hline 8) & $\begin{array}{l}\text { The (not cat) had asked me its food } \\
\text { before it was (not) left at the house. }\end{array}$ & Negative & Positive & Negative & Eighth \\
\hline
\end{tabular}

$$
\boldsymbol{M}(\hat{\boldsymbol{r}}(\phi, \theta))=(14+\sin \phi \cos \theta) \hat{\boldsymbol{i}}+(2+\sin \phi \sin \theta) \hat{\boldsymbol{j}}+\mathrm{e}^{\cos \phi} \hat{\boldsymbol{k}}+(1) \hat{\boldsymbol{i}}
$$

The value of $\boldsymbol{r}_{\phi} \times \boldsymbol{r}_{\theta}$ can be estimated using Equation (14). Therefore: $\boldsymbol{M}(\hat{\boldsymbol{r}}(\phi, \theta)) \cdot\left(\boldsymbol{r}_{\phi} \times \boldsymbol{r}_{\theta}\right)$ $=\left(14 \sin ^{2} \phi \cos \theta+\sin ^{3} \phi \cos ^{2} \theta\right)+\left(2 \sin ^{2} \phi \sin \theta+\sin ^{3} \phi \sin ^{2} \theta\right)+\left(\sin \phi \cos \phi \mathrm{e}^{\cos \phi}\right)$

Then, the value of (MF) will be:

$$
\begin{aligned}
& \mathrm{MF}=\iint_{D} \boldsymbol{M} \cdot\left(\boldsymbol{r}_{\phi} \times \boldsymbol{r}_{\theta}\right) \mathrm{d} A \\
&= \int_{0}^{2 \pi} \int_{0}^{\pi}\left[\left(14 \sin ^{2} \phi \cos \theta+\sin ^{3} \phi \cos ^{2} \theta\right)\right. \\
&\left.+\left(2 \sin ^{2} \phi \sin \theta+\sin ^{3} \phi \sin ^{2} \theta\right)+\left(\sin \phi \cos \phi \mathrm{e}^{\cos \theta}\right)\right] \mathrm{d} \phi \mathrm{d} \theta \\
&= \int_{0}^{\pi} 14 \sin ^{2} \phi \mathrm{d} \phi \int_{0}^{2 \pi} \cos \theta \mathrm{d} \theta+\int_{0}^{\pi} \sin ^{3} \phi \mathrm{d} \phi \int_{0}^{2 \pi} \cos ^{2} \theta \mathrm{d} \theta+\int_{0}^{\pi} 2 \sin ^{2} \phi \mathrm{d} \phi \int_{0}^{2 \pi} \sin \theta \mathrm{d} \theta \\
&+\int_{0}^{\pi} \sin ^{3} \phi \mathrm{d} \phi \int_{0}^{2 \pi} \sin ^{2} \theta \mathrm{d} \theta+\int_{0}^{\pi} \sin \phi \cos \phi \mathrm{e}^{\cos \phi} \mathrm{d} \phi \int_{0}^{2 \pi} \mathrm{d} \theta \\
&=\left(7\left[\phi-\frac{1}{2} \sin 2 \phi\right]_{0}^{\pi}[\sin \theta]_{0}^{2 \pi}\right)+\left[\left(\frac{1}{3} \cos ^{3} \phi\right)-\cos \phi\right]_{0}^{\pi}\left[\frac{1}{2} \theta+\frac{1}{4} \sin 2 \theta\right]_{0}^{2 \pi} \\
& \quad-\left[\phi-\frac{1}{2} \sin 2 \phi\right]_{0}^{\pi}[\cos \theta]_{0}^{2 \pi} \cdot\left(\frac{1}{2}\right)\left[\theta-\frac{1}{2} \sin 2 \theta\right]_{0}^{2 \pi}+\left[\cos \phi \mathrm{e}^{\cos \phi}-\mathrm{e}^{\cos \phi}\right]_{0}^{\pi} \\
&=7(\pi-0)(0)+[1 / 3(-1)+1][\pi+0-[\pi-0][1]+[0-0][\pi-0]-2 / \mathrm{e}] \\
&=2 / 3-2 / \mathrm{e}
\end{aligned}
$$

\section{The Explanation and the Interpretation of a Text}

There is a real confusion linguistically between the two terms: "Explanation" and "Interpretation". In general, the term "explanation" has been used as a descrip- 
tion of facts, hypothesis, or even an individual opinion of a speaker. This can be missed-described from the speaker sometimes or even cannot be accepted from the audience for different reasons; Ruben (1992). The mode of thinking also plays an important role in the explanation and makes it impossible to separate the speaker away from the situation and the world or the culture around him, Castillo (2013).

The term "interpretation" appears to be bigger or greater to include the analysis of a text deeply to identify the intention of the speaker or the writer during the speaking process or the writing process. Ricoeur (1976) stated that the term "interpretation" is a complicated process including two steps or two functions. The first is the identification function of the language or the discourse symbols in an event, while the second function is the prediction process which includes an extrapolation along the space of a text to approach the meaning beyond it.

Glasersfeld (1983) concluded that we can come across the term interpretation not only in literary studies. It has been used in music, law, acting, translating, psychology, computer science, mathematics and even architecture studies. In his opinion the interpretation term design or visualize an activity or expose its result. Furthermore, the interpreter has to be able to combine between the conceptual structures and the symbolic representation.

Jeff (2006) in his review of the meaning theory used the term interpretation as a differential tool to choose between different logically identified semantic cases. He is strongly supporting the idea of Lewis (1970) by having two types of theory of meaning: The first of them the description of the language structure and grammar symbols abstractly to address their world aspects. The second is the estimation of the psychological and sociological facts whereby a specific semantic system was used by an individual or a group of individuals in the community body.

We can clearly see that some authors in different fields are frequently using the two terms "explanation" and "interpretation" as a synonyms to express the semantic meaning of a specific text or a speech. A few authors could be able to differentiate between two levels of meaning and they succeeded, to some extent, to apply the term explanation to the identification of language symbols and its structure meaning level. The other level (= the deep meaning) needs an analytical, psychological, philosophical and logical experience to combine and extrapolate between the hidden bonds of the semantic symbols usage and the significance beyond that.

In recent publications, the second type was not given a specific name, unfortunately, but we can clearly see that in older publications particularly those which were dealing with the holly books studies, the interpretation was introduced back a day to describe the diving deeply process into the bottoms of the scriptures to capture a higher dimension meaning, c.f. Ibn Arabi (1165-1240).

In the present model we can recognize two types of meaning. The first which we call the explanation $(E)$ is related to the direct and indirect identification of 
the language symbols and structures in four dimensions. Equation (16) which is the general form of Equation (9) represents the explanation term $(E)$ as follows:

$$
\boldsymbol{E}=\boldsymbol{M}_{i, j, k, l}=(a x+b) \hat{\boldsymbol{i}}+(c y+d) \hat{\boldsymbol{j}}+\left(f \mathrm{e}^{z}\right) \hat{\boldsymbol{k}}+q \hat{\boldsymbol{l}}
$$

where $a, b, c, d, f, q$ are real numbers.

The reason beyond that nomination is related to the ability of humans regardless of their ages to use metaphors and understand them. For instance on one hand, kids can use the figurative speech and can communicate with it in the dialect lower form of any natural language. On the other hand, the adults can do the same in higher classic form and even in higher dialect level of a certain natural language.

The second type which we call interpretation of meaning is the deep meaning. That type including other dimensions such as but not limited: the metaphysical power, the psychological state of the speaker-writer, the integration experience of the interpreter and the ability to penetrate the space of the text or a speech. This kind of meaning will open a complete reading to the text especially those contribute substantial values in human life.

The interpretation term $(I)$ of a text can be written mathematically as:

$$
\boldsymbol{I}=\sum_{i=1}^{n} f_{i} \hat{\boldsymbol{e}}_{i}=f_{1} \hat{\boldsymbol{e}}_{1}+f_{2} \hat{\boldsymbol{e}}_{2}+K f_{n} \hat{\boldsymbol{e}}_{n}
$$

where $f_{1}, f_{2}, f_{3}, K, f_{n}$ are the component functions in the direction of the unit vectors $\hat{\boldsymbol{e}}_{1}, \hat{\boldsymbol{e}}_{2}, \hat{\boldsymbol{e}}_{3}, \hat{\boldsymbol{k}}, \hat{\boldsymbol{e}}_{n}$.

It is clear that the relationship between the explanation term $E$ and the interpretation term $\boldsymbol{I}$ is $\boldsymbol{E} \subset \boldsymbol{I}$.Therefore, the interpretation of a text includes the explanation and strengthens it. The explanation $E$ is the meaning function in four-dimensions or lower, while the interpretation $I$ is the meaning function in higher dimensions. This will solve the dilemma of the interpretation of the Holly books. The interpretations of the scriptures are suffering from a discrepancy case. Some of the interpreters have gone far away from the explicit domain of the explanation by the excuse of dealing with a deep reading of the scripture text. This reading can match or even can contradict the explanation of the same text which is skeptically unacceptable.

\section{Discussion and Conclusion}

The meaning function captured the minds of a huge number of scholars in different fields all over the human intellectual history. There are a lot of concepts and procedural models that tried to explain the mechanism of the communication process between the "speaker-writer" and the "audience-reader". There was an overwhelming between the procedural linguistic view and the psychological, metaphysical, and philosophical aspects of a natural language. This held the computational analysis of the target language numerically due to the lack of distinction zones between the procedural attempt, the induction process and the metaphysical approach constructing a specific human language. 
The authors introduced in this paper their new approach of the meaning function as a vector valued function of four-dimensional components. The function was mathematically formulated based on the vector function analysis considering the speech process as a vector field. The one-dimensional approach was represented by the dictionary meaning of words in any human language which came by convention through generations inside the body of a linguistic society. The presence of the sentence structure and the intonation symbols in the text added a second variable and pushed the meaning function toward the two-dimensional concept.

The rhetorical tools came along and reformed the two-dimensional concept to be a three-dimensional attempt. The rhetorical are representing the amplitudes of the two-dimensional model of the meaning function. The model becomes complicated and appears to be unconformable with the nature of some rhetorical tools such as metaphors and metonymies. This consequently leads to an obligation case which is the presence of higher-dimension due to the existence of the metaphors or the metonymies in the speech.

The unconformable irregularities of the meaning model were taken care and the meaning approach reappears consistent by the four-dimensional view. When the $x, y$, and $z$ components of the meaning function in relation to $X, Y$, and $Z$ Cartesian axes respectively approach infinity; the imaginative power will be close enough to contact the three dimensional space of the meaning function introducing the fourth component $q(t)$ in the $S$ direction, Equation (8).

The two terms "explanation" and "interpretation" became unclear recently due to the lack of the distinctive zones between the conceptual procedural aspect and the way meta-physicists, philosophers and psychology researchers approach the meaning. The authors formulated the model on the basis of having two types of meaning. The first which we call the explanation is related to the direct identification of the language symbols and structures in four dimensions.

The second type which we call interpretation of meaning is the deep meaning. That type relies on other dimensions including but not limited: the metaphysical power, the psychological state of the speaker-writer, the integration experience of the interpreter and the ability to penetrate the space of the text or a speech. This kind of meaning will open a complete reading to the text especially those contributing important interests in human life.

One can say that there is no existence of time in this model, while it has been believed to be a physical dimension which must be included in everything related to matter and any derived activity based on it in the universe. We argue that time, in the text or in a speech, is appearing in the structural component. In the dictionary meaning, time can be captured in some words like verbs. This capture can be interpreted as an intersection between time in higher dimension and the lower dimensions of the meaning function components synchronous to their formation by the speaker or the writer.

In addition, the text and its space domain now can be easily constructed as a combination of vectors and the properties of the latter will help in analyzing the 
text computationally. This in turn will lead to outstanding applications such as improving digital translation quality and enhancing the differential interpretation of serious events in all life activity fields. The literature issues can be evaluated by a standard measuring tool based on the current model.

The stock market reports and even the defense background intelligence information authorities can take advantage of that model. Now encoding of a report (text) will achieve advanced level by adding and analyzing the figurative speech part. The decision makers will stand on a stable-ground instead of guessing in making their vital and crucial decisions. In addition, the accuracy of the IQ intelligent test for intelligence will be increased to reflect credible reality intelligence levels. The current IQ test method which has been failed to match and to draw a general attempt in estimating the creative intelligence of humans should be upgraded.

Furthermore, the higher-defense scientists can be able to observe and classify individuals in early stage according to their ability of practical intelligence or creative intelligence and assign them the suitable positions. Not only that, the service members also can be tracked through their essays or even through their official reports. Repeated inapplicable task missions or orders reflect deficiency in knowledge or mental illness problems. These problems have to be addressed and removed quickly from the system for a successful consistence dynamic flowing of the organization. Our model introduces a numerical valuable way facilitating this job. The current model in this paper is easier when it is compared with the very difficult old method; which consuming money and time as well as delaying strategic plans in emergency cases.

Finally, robots digital speaking industry would show a huge jump if our meaning model is taking into consideration. The ability of making advanced robots and call centers which can communicate completely with humans will be ordinary objective rather than what it is. Based on what we mentioned, the existing model can be a start up branch in both literary and technology fields inspiring imaginative ideas to decorate its shiny bright temple in the starry carpet of human intellectual activity.

\section{Acknowledgements}

The authors are expressing their sincere gratitude to Lutheran Metropolitan Ministry staff and personnel for their continuous financial and logistic support. The authors are highly thankful for the City of Cleveland in Ohio, USA who put its facilities and equipments on demand to support this work. The authors mention the mutual cooperation of Cleveland State University (CSU), Cleveland, $\mathrm{OH}$.

The authors truly cannot deny the effective role of the staff of the Department of Mission and Bureau in the Ministry of Higher Education, Cairo, Egypt for their educational fund and the English language preparation.

The authors are indebted to Dr. Raafat Abu Zaid, Cairo, Egypt for his fruitful 
mathematical comments on an early draft of this paper. The authors are expressing their deepest appreciation to Dr. Mark Giuliano, the senior pastor at Stone Church in Cleveland, OH, USA for his support and review. The authors appreciate the advice and support of both Mr. Burt Salzman, the owner of Dave's Supermarket in Ohio, USA and Carnegie Auto Detail Shop staff in Cleveland.

The authors wish the best for Marcie Goodman, the executive director of Cleveland International Film Festival, Cleveland, $\mathrm{OH}$, for her sincere effort and her critical notes. Last but not least, the authors want to extend their appreciation to those who could not be mentioned here but here well played their role to support this work till the end.

\section{References}

Castillo, J. M. (2013). Modes of Thinking in Language Study. European Scientific Journal, 4, 421-431.

Cook, P., \& Stevenson, S. (2010). Automatically Identifying the Source Words of Lexical Blends in English. Computational Linguistics, 36, 129-149.

https://doi.org/10.1162/coli.2010.36.1.36104

Fass, D. (1991). A Method for Discriminating Metonymy and Metaphor by Computer. Journal of Computational Linguistics, 17, 49-90.

Fauconnier, G., \& Turner, M. (2002). The Way We Think: Conceptual Blending and the Mind's Hidden Complexities (446 p). New York: Basic Books Pub.

Gagliano, A., Paul, E., Booten, K., \& Hearst, M. (2016). Intersecting Word Vectors to Take Figurative Language to New Heights. Proceedings of the Fifth Workshop on Computational Linguistics for Literature, San Diego, 1 June 2016, 20-31. https://doi.org/10.18653/v1/W16-0203

Glasersfeld, E. (1983). On the Concept of Interpretation. Poetics, 12, 207-218. https://doi.org/10.1016/0304-422X(83)90028-1

Hirtle, W. H. (1985). Linguistics and the Dimensions of Language: An Overview of Guillaume's Theory. Lingua, 67, 65-83. https://doi.org/10.1016/0024-3841(85)90013-0

Ibn, A. (1165-1240) (1911). Al-futuhat Al-makkiyyah (Vol. 1). Cairo: Bulaq Pub.

Jeff, S. (2006). Truth Theories, Translation Manuals, and Theories of Meaning. Linguistics and Philosophy, 29, 487-505.

Lakoff, G., \& Turner, M. (1989). More than Cool Reason: A Field Guide to Poetic Metaphor (273 p.). Chicago, IL: University of Chicago Press. https://doi.org/10.7208/chicago/9780226470986.001.0001

Leon, S. (2006). Linear Algebra with Applications-7E (523 p.). Upper Saddle River, NJ: Pearson Prentice Hall.

Lewis, D. (1970). General Semantics Syntheses (Vol. 22, pp. 18-67).

Ricoeur, P. (1976). Interpretation Theory: Discourse and the Surplus of Meaning (107 p.). Fort Worth, TX: Texas Christian University Press.

Ruben, D. (1992). Explaining Explanation (265 p.). Abingdon-on-Thames: Routledge Inc. Science Wiki (2017). Fandom. http://el.science.wikia.com/wiki

Stewart, J. (1999). Calculus-4E (1185 p.). Grove, CA: Brooks Cole Publishing Co. 